\title{
A mechanistic view of mitochondrial death decision pores
}

\section{J.E. Belizário ${ }^{1}$, J. Alves ${ }^{1}$, J.M. Occhiucci ${ }^{1}$, \\ M. Garay-Malpartida ${ }^{1}$ and $\mathrm{A}$. Sesso ${ }^{2}$}

\author{
1Departamento de Farmacologia, Instituto de Ciências Biomédicas, \\ ${ }^{2}$ Laboratório de Imunopatologia, Instituto de Medicina Tropical, \\ Universidade de São Paulo, São Paulo, SP, Brasil
}

\author{
Correspondence \\ J.E. Belizário \\ Departamento de Farmacologia \\ ICB, USP \\ Av. Lineu Prestes, 1524 \\ 05508-900 São Paulo, SP \\ Brasil \\ Fax: +55-11-3091-7322 \\ E-mail: jebeliza@usp.br \\ Research supported by FAPESP \\ (No. 01/01000-7), CAPES and CNPq. \\ $\ldots \ldots \ldots \ldots \ldots \ldots \ldots$
}

Received October 27, 2005 Accepted February 16, 2007
Abstract

Mitochondria increase their outer and inner membrane permeability to solutes, protons and metabolites in response to a variety of extrinsic and intrinsic signaling events. The maintenance of cellular and intraorganelle ionic homeostasis, particularly for $\mathrm{Ca}^{2+}$, can determine cell survival or death. Mitochondrial death decision is centered on two processes: inner membrane permeabilization, such as that promoted by the mitochondrial permeability transition pore, formed across inner membranes when $\mathrm{Ca}^{2+}$ reaches a critical threshold, and mitochondrial outer membrane permeabilization, in which the pro-apoptotic proteins BID, BAX, and BAK play active roles. Membrane permeabilization leads to the release of apoptogenic proteins: cytochrome $c$, apoptosisinducing factor, Smac/Diablo, HtrA2/Omi, and endonuclease G. Cytochrome $c$ initiates the proteolytic activation of caspases, which in turn cleave hundreds of proteins to produce the morphological and biochemical changes of apoptosis. Voltage-dependent anion channel, cyclophilin D, adenine nucleotide translocase, and the pro-apoptotic proteins BID, BAX, and BAK may be part of the molecular composition of membrane pores leading to mitochondrial permeabilization, but this remains a central question to be resolved. Other transporting pores and channels, including the ceramide channel, the mitochondrial apoptosis-induced channel, as well as a non-specific outer membrane rupture may also be potential release pathways for these apoptogenic factors. In this review, we discuss the mechanistic models by which reactive oxygen species and caspases, via structural and conformational changes of membrane lipids and proteins, promote conditions for inner/outer membrane permeabilization, which may be followed by either opening of pores or a rupture of the outer mitochondrial membrane.

\section{Introduction}

Recent studies have identified, among the variety of ways by which cells have been reported to die, three major forms of cell death: apoptosis, necrosis and autophagy.

\section{Key words}

- Mitochondrial outer membrane permeabilization

- Permeability transition pore

- Cytochrome c

- Reactive oxygen species

- Caspases

- BCL-2 
adult life (1). Research conducted over the past few years has provided evidence that diverse extracellular and intracellular signaling events within apoptosis converge toward mitochondria (2-7). In mitochondrially mediated apoptosis, inner and/or outer mitochondrial membranes undergo a permeabilization process that causes the release and redistribution of small ions, solutes and metabolites, as well as of cytochrome $c$, a 14-kDa protein that functions as an electron carrier in the mitochondrial respiratory chain. In mammals, cytochrome $c$ is a necessary co-factor for activation of caspase-9, a member of the protease family that coordinates the biochemical and morphological events of apoptosis (1-7).

It is presumed that cytochrome $c$ and other apoptogenic factors, including Smac/ Diablo, HtrA2/Omi and apoptosis-inducing factor (AIF), are released as a result of the opening of large non-selective pores known as "permeability transition pores" (PTP) $(1,7)$ or through a proteolipid pore spanning only the outer mitochondrial membrane $(2,6)$. In vitro and in vivo studies have demonstrated that cytoplasmic and mitochondrial protein complexes and the membrane lipid environment are involved in the formation and function of these putative pores. Proteins of the BCL-2 family with pro-apoptotic properties such as BID, BAX, and BAK may cooperate in the formation of these pores, together with the major mitochondrial voltage-dependent anion channel known as VDAC, adenylate nucleotide translocase (ANT) and cyclophilin D (Cyclo D). There is also evidence that the release of apoptogenic factors could occur through a putative channel named mitochondrial apoptosis-inducing channel or MAC (8) and through a large channel containing ceramide lipids, i.e., the ceramide channel (9). A rupture of the outer mitochondrial membrane could also work as a pathway for the release of apoptogenic proteins (10).

The formation of these pores in the inner and outer membrane depends on a variety of bioenergetic, membrane transport and redox conditions that ultimately lead to major changes in the structure of mitochondrial proteins and lipids. Reactive oxygen species (ROS) generated inside and outside the mitochondria are important promoters of chemical modification and conformational changes of membrane polypeptides and lipids $(11,12)$. Caspases are cysteine proteases that play a central role in intracellular proteolytic pathways by inducing structural and functional changes in various vital proteins involved in apoptosis and various other nonapoptotic processes such as inflammation, cell cycle and differentiation $(13,14)$. Various procaspases and active caspases, including $-3,-7,-8$, and -9 , are localized in, or translocate to, mitochondria during apoptosis, perhaps controlling the permeabilization of this organelle (15). More recently, the critical involvement of caspases in mitochondrially mediated apoptosis has been demonstrated using mice lacking caspase-3 and -7 (16). The key concept in this model is that certain critical regulators located in the outer and inner mitochondrial membranes or within the matrix could act as caspase substrates. Thus, the point-specific cleavage of one site of substrate proteins could be a commitment step toward membrane permeabilization.

\section{Reactive oxygen species-induced mitochondrial structural modifications and permeability transition pores}

All mammals use $\mathrm{O}_{2}$ for energy production $(11,12)$. Oxidation is the loss of an electron by a substance. Under normal metabolic conditions, electron-transporting complexes I, II, III, and IV plus a non-redox $\mathrm{H}^{+-}$ translocating complex, ATP synthase (also called complex $\mathrm{V}, \mathrm{F}_{0} \mathrm{~F}_{1}$-ATP synthase), together with co-enzyme $\mathrm{Q}$ and cytochrome $c$, carry out oxidative phosphorylation. Com- 
plex II is completely encoded by the nucleus, whereas complexes I, III and IV are encoded by nuclear and mitochondrial DNA. The respiratory enzyme complexes transfer electrons $\left(\mathrm{H}^{-} \rightarrow \mathrm{H}^{+}+2 \mathrm{e}\right)$ from the reducing equivalents NADH or $\mathrm{FADH}_{2}$ to $\mathrm{O}_{2}$, while transporting protons across the inner mitochondrial membrane. The total proton-motive force across the inner mitochondrial membrane is the sum of a large force derived from the mitochondrial membrane electrical potential $(\rho \Psi \mathrm{m})$ and a smaller force derived from the $\mathrm{H}^{+}$concentration gradient $(\Delta \mathrm{pH})$. This proton-motive force is used to drive protons from the intermembrane space into the matrix through ATP synthase, a transmembrane protein complex that uses the energy of $\mathrm{H}^{+}$flow to synthesize ATP from ADP and Pi. This electrochemical proton gradient is also required to import mitochondrial proteins and to regulate metabolite transport across the mitochondrial membrane $(11,12)$.

A small percentage of the total $\mathrm{O}_{2}$ consumed by the mitochondrial electron transport chain in healthy tissues becomes ROS, such as superoxide $\left(\mathrm{O}_{2}{ }^{-}\right)$, hydrogen peroxide $\left(\mathrm{H}_{2} \mathrm{O}_{2}\right)$ and hydroxyl radical $\left(\mathrm{OH}^{-}\right)$ $(11,12)$. This ROS production occurs primarily in complex I (NADH dehydrogenase) and complex III (ubiquinone-cytochrome $c$ reductase). $\mathrm{O}_{2}$ itself is also a free radical because is has two unpaired electrons in its outer orbit which make it reactive. The two unpaired electrons in $\mathrm{O}_{2}$ have parallel spins, which means that $\mathrm{O}_{2}$ can only oxidize another molecule by accepting a pair of electrons that have antiparallel spins or one electron at a time. Superoxide has one electron more than $\mathrm{O}_{2}$. Since only one electron is unpaired in $\mathrm{O}_{2} \cdot-$, superoxide is more reactive than $\mathrm{O}_{2}$. However, $\mathrm{O}_{2}{ }^{-{ }^{-}}$is still not a very reactive radical, and, in the presence of $\mathrm{H}^{+}$or $\mathrm{HO}_{2} \cdot$, can reduce $\mathrm{O}_{2} \cdot{ }^{--}$to $\mathrm{H}_{2} \mathrm{O}_{2}$ or be oxidized to $\mathrm{O}_{2}(12)$.

$\mathrm{H}_{2} \mathrm{O}_{2}$ is more stable than $\mathrm{O}_{2}{ }^{--}$and quickly diffuses across membranes. In the presence of iron in the ferrous form $\left(\mathrm{Fe}^{2+}\right), \mathrm{H}_{2} \mathrm{O}_{2}$ can be reduced to the highly reactive $\mathrm{OH}^{\cdot}$ radical through the Fenton reaction. $\mathrm{O}_{2} \cdot{ }^{--}$can react with reactive nitrogen species, such as nitric oxide and nitrogen dioxide $\left(\mathrm{NO}_{2}\right)$ to form peroxynitrite $\left(\mathrm{ONOO}^{-}\right)$. Both the oxygen- and nitric oxide-based radicals attack DNA, proteins, lipids, and carbohydrates to produce DNA strand breaks, protein oxidation and lipid peroxidation. The amino acids tyrosine, histidine, arginine, lysine, and proline are particularly vulnerable to ROS modification, which translates to gain or loss of receptor activity, enzyme function and signal transduction pathways $(12,17,18)$.

Efficient biochemical and bioenergetic mechanisms aimed at controlling ROS production and ensuring its removal have emerged $(11,12)$. The first antioxidant enzyme described is superoxide dismutase (SOD). This enzyme catalyzes the reaction that converts two $\mathrm{O}_{2}{ }^{--}$and two $\mathrm{H}^{+}$to $\mathrm{H}_{2} \mathrm{O}_{2}$ and $\mathrm{O}_{2}$. Three isoforms of this enzyme have been well characterized; $\mathrm{SOD}_{1}$, a copper/ zinc $(\mathrm{Cu} / \mathrm{Zn})$ isoform present in the cytosol; $\mathrm{SOD}_{2}$, a manganese $(\mathrm{Mn})$ isoform present in mitochondria, and $\mathrm{SOD}_{3}$, a $\mathrm{Cu} / \mathrm{Zn}$ isoform present in the extracellular space. Cysteine, glutathione, ascorbic acid (vitamin C), and $\alpha$-tocopherol (vitamin E) are other important antioxidants that limit injuries induced by $\operatorname{ROS}(11,12)$.

At the physiological level, both $\mathrm{H}_{2} \mathrm{O}_{2}$ and superoxide $\left(\mathrm{O}_{2}{ }^{--}\right)$can act as second messengers in cellular signaling that leads to the activation and inactivation of ion channels, receptors, enzymes (kinases and phosphatases), and transcription factors $(12,17,18)$. However, above a threshold level, ROS can randomly attack macromolecules, interfering with vital pathways to cause cell death either by apoptosis or necrosis $(19,20)$. Various mechanisms have been explored to explain how ROS activate cell death signaling pathways $(19,20)$. Earlier studies have shown that $\mathrm{OH} \cdot$ can oxidize thiol $(-\mathrm{SH})$ groups of 
sensor proteins, activating the PTP (21). The PTP is considered to function as the point of no return for both apoptosis and necrosis $(20,21)$. Under a variety of experimental conditions, $\mathrm{Ca}^{2+}$ is a powerful co-activator of PTP in response to oxidative stress $(19,21)$. The opening and operation of PTP can be prevented by cyclosporine A, a cyclic peptide that binds to Cyclo D, its mitochondrial matrix molecular receptor. However, there are situations in which the inhibitory effect is partial, transient or null. In addition, several studies have shown that cyclosporines are non-selective inhibitors of seven transmembrane helix G protein-coupled receptors, plasma membrane ion channels and $\mathrm{ABC}$ transporters $(7,21)$.

Despite extensive research in many laboratories, it has been difficult to isolate and identify the components of PTP, as well as the extent of contribution of ROS to its formation and operation $(7,20,21)$. Previous studies have suggested that PTP is a supramolecular complex which may contain or be regulated by ANT, VDAC, Cyclo D, and the peripheral benzodiazepine receptor $(7,21)$. While in some pathways the transient opening of PTP is viewed as the first step to apoptosis, in many others, apoptosis can be independent of this process $(2,4-6)$. Some investigators have also considered the possibility that the onset of PTP is associated with the transition from apoptosis to necrosis $(20,21)$.

\section{Caspase activation}

Recent findings have provided a new framework for understanding the upstream and downstream events of mitochondrial dysfunction and the critical roles of proteins of the BCL-2 and caspase family in apoptosis $(2,4-6)$.

Caspases constitute a family of evolutionarily conserved aspartate-specific cysteine-dependent proteases that have major roles in apoptotic and inflammatory path- ways $(13,14,22)$. They are synthesized as pro-enzymes containing a prodomain of variable length that is attached to the enzymatic subunits. Upon proteolytic activation and release of the pro-domain, the subunits undergo a conformational change to form the active enzyme. Caspases can be classified into two groups: "initiator caspases" contain a large prodomain in contrast to "executioner caspases" which are characterized by a small prodomain. Initiator caspases harbor protein-protein interaction modules: the caspase recruitment domain (CARD) in caspases-1, -2, -4, -5, -9, -11, -12, and death effector domain in caspases- 8 and -10 . These motifs are characterized by the presence of six or seven anti-parallel amphipathic $\alpha$ helices, which allow the recruitment of other signaling molecules or adaptor molecules in large protein complexes, thereby initiating apoptotic or inflammatory signaling pathways (13).

Caspase activation can occur secondarily to triggering the extrinsic or intrinsic pathways of apoptosis $(1,2)$. The extrinsic pathway of apoptosis is triggered by cytokines in the CD95/Fas/APO-1, TNF and TRAIL families (13) upon their binding to membrane cell death receptors. An intracellular adaptor protein, called Fas-associated protein with a death domain or TNF receptor associated with a death domain then recruits the initiator procaspase-8, via its death effector domain, to a death-inducing signaling complex. This complex facilitates the processing and full activation of this enzyme that, in turn, promotes the cleavage of specific substrates and executioner caspase-3.

A pivotal event in the intrinsic pathway of apoptosis is the release of cytochrome $c$ from the mitochondrial intermembrane space. Once into the cytosol, holo-cytochrome $c$ (that is formed within mitochondria) readily associates with the C-terminal region of apoptotic protease-activating factor (Apaf-1) that contains 12-13 WD40 repeats. This interaction facilitates the binding of dATP with 
Apaf-1 and exposes its N-terminal CARD, which can now oligomerize and form a procaspase-9-activating platform. The resulting oligomeric Apaf-1 complex is able to recruit several inactive procaspase-9 molecules through heterotypic CARD-CARD interactions to form the so-called apoptosome. The apoptosome then activates initiator caspases $(13,23)$.

Cytoplasmic proteins named inhibitors of apoptosis prevent unintended caspase-9 activation (24). These proteins bind to and inhibit the newly generated active $\mathrm{N}$ terminus of caspase-9. This inhibition is relieved after the release of inhibitors of apoptosisantagonizing proteins Smac/Diablo and HtrA2/Omi from mitochondria $(25,28)$. These two proteins work as second level regulators of the apoptotic process. Mitochondria also release two proteins that have DNA endonuclease activity: endonuclease $\mathrm{G}$ (Endo G) and the AIF, a 57-kDa flavoprotein (28). Because of differences in size and shape as well the kinetics of diffusion, it is presumed that a distinct pore or even a rupture of the outer mitochondrial membrane allows for the release of these two proteins $(25,28)$.

Activated caspases promote proteolytic cleavage of various vital proteins during apoptosis and non-apoptosis processes, which result in either activation or inactivation of their substrates $(14,15,22)$. Cleavage-induced activation can lead to differential regulation, stabilization, protein complex formation, and special localization for the protein or its fragments (22). In some cases, a first cut by caspases unleashes additional cleavage sites for other proteases. In other cases, cleavage allows for structural changes and exposure of previously hidden structures (15). More than 300 proteins have been characterized as caspase substrates (15). Plasma membrane receptors and structural, regulatory cytosolic and nuclear proteins are preferred targets for executioner caspases such as caspase-3, -6, and -7 (15).

\section{Mitochondrial outer membrane permeabilization by BCL-2 family proteins}

BCL-2 family members are of particular interest among substrates that are activated by caspases during the two pathways of apoptosis $(4,5)$. The BCL-2 family consists of multidomain members like BAX, BOK and BAK and the $\mathrm{BH} 3$-only group of pro-apoptotic members, including BID, BAD, BIK, and BIM. The anti-apoptotic members BCL-2 and BCL$\mathrm{x}_{\mathrm{L}}$ have $4 \mathrm{BH}$ domains (BH1-4). All of these proteins have the ability to bind to membranes and form, predominantly under non-physiological conditions, ion-conducting channels in synthetic membranes $(4,5)$.

Caspase- 8 promotes the cleavage of BID to form its C-terminal truncation, tBID, which moves to the outer mitochondrial membrane and induces the permeabilization process named mitochondrial outer membrane permeabilization (MOMP) (2), allowing the formation of an outer membrane-spanning pore through which cytochrome $c$ is released $(4,5)$. Studies using BAX and BAK doubly deficient cells and knockout mice have shown that MOMP by BH3-only molecules such as $\mathrm{BID}$ and BIM requires BAX and BAK $(2,4-$ 6 ), each of which can form homo-oligomers and hetero-oligomers in the outer mitochondrial membrane (2,4-6).

The pro-survival proteins BCL-2 and BCL- $\mathrm{x}_{\mathrm{L}}$ prevent the release of cytochrome $c$ from mitochondria induced by many apoptotic signals (4). It seems that BCL-2 may adapt or regulate mitochondrial homeostasis through a combination of different effects, including modulating the formation of ROS, intracellular acidification and proton fluxes in the mitochondria (3). The ability to interact and sequester tBID away from BAX and BAK proteins has also being considered of fundamental importance for their pro-survival effects (4). Interestingly, BCL-2 and $\mathrm{BCL}-\mathrm{x}_{\mathrm{L}}$ are also cleaved by caspases during apoptosis when the $\mathrm{N}$-terminal $\mathrm{BH} 4$ domain 
is released, enabling the new fragment to promote apoptosis (4-6).

In healthy cells, several BCL-2 members, including BCL-2 and BCL- $\mathrm{x}_{\mathrm{L}}$ are inserted not only into the outer membrane of mitochondria but also into the endoplasmic reticulum (ER). Overexpression of BCL-2 reduces ER $\left(\mathrm{Ca}^{2+}\right)$ levels and this exerts a protective effect against some apoptotic responses (29). The pro-apoptotic members BAX and BAK promote $\mathrm{Ca}^{2+}$ mobilization from the ER to mitochondria (29). Thus, these proteins may operate as regulators of $\mathrm{ER} \mathrm{Ca}^{2+}$ concentrations and modulate the propagation of $\mathrm{Ca}^{2+}$ waves into mitochondria (29).

\section{BCL-2 family proteins and voltage- dependent anion channel activity}

The most common pathway for the translocation of metabolites through the outer membrane under physiological conditions is the VDAC (30-32). Three isoforms (VDAC1, VDAC2 and VDAC3) of molecular mass around $30 \mathrm{kDa}$ have been identified in multicellular organisms (31). The archetypal VDAC1 is a large diameter $\beta$ barrel structure composed of one $\alpha$ helix and $13 \beta$ strands whose aqueous channel $(2.5-3 \mathrm{~nm})$ adopts multiple conductance states with special selectivity between cations and anions (30). VDAC seems to serve as an important docking site for cytosolic mitochondrial intermembrane space and inner membrane proteins such as ANT, hexokinase, Cyclo D, creatine kinase, glycerol kinase, and the peripheral benzodiazepine receptor, as well as BCL-2 family proteins $(30,32)$. However, caution should be exercised in considering the various reports of protein association with VDAC, given its large excess over other proteins in the outer mitochondrial membrane.

VDAC exists in an open configuration that permits the free exchange of most metabolites of molecular mass up to $5 \mathrm{kDa}$ in size. At a voltage smaller than $30 \mathrm{mV}$, the pore has a diameter of $2.5-3 \mathrm{~nm}$ and is in the anion, high conducting state, referred to as the open state. This open state permits the passage of $\mathrm{ATP}^{-4}, \mathrm{HPO}_{4}^{-2}$, succinate ${ }^{-2}$, and other negatively charged molecules. Above $30 \mathrm{mV}$, the diameter decreases to $\sim 1.8 \mathrm{~nm}$, the conductance decreases to $2 \mathrm{nS}$ and selectivity changes to cations. In this closed state, VDAC favors the flux of small cations such as $\mathrm{Ca}^{2+}, \mathrm{K}^{+}$, and $\mathrm{Na}^{+}$, but is impermeable to the respiratory substrates ATP and ADP (30). It is important to mention that the VDAC conductance states measured in a planar lipid membrane system vary depending on the salt and lipid concentrations $(30,32)$.

The idea that VDAC, ANT and Cyclo D are the core components of the permeability transition pore $(33,34)$ has been further explored recently using gene knockout and shRNA strategies (21). Silencing VDAC1 expression diminished cell growth and mitochondrial ATP synthesis. On the other hand, the basic properties of the PTP did not change in VDAC1 $1^{--}$mitochondria. Thus, the participation of VDAC in PTP composition remains an open question $(35,36)$.

It is now becoming clearer how the BCL2 family proteins interfere with the channel activity of VDAC in vitro and in vivo $(3,32)$. Experiments with VDAC channels reconstituted into the lipid matrix demonstrated that anti-apoptotic BCL- $\mathrm{x}_{\mathrm{L}}$ promotes the maintenance of VDAC in a physiological open state $(3,32)$. One study has shown that proapoptotic tBID induces VDAC closure, while BAX does not affect the conductance of this channel (32). tBID could affect VDAC conductance indirectly through the lipid environment surrounding VDAC (32) and not through a direct physical interaction $(33,34)$. VDAC in the closed state favors permeability to cations, like $\mathrm{Ca}^{2+}, \mathrm{K}^{+}$and $\mathrm{Na}^{+}(30)$.

\section{BCL-2 family proteins and the mitochondrial apoptosis-induced channel}

Recent reports have suggested that a new 
channel, known as MAC, is a pathway for cytochrome $c$ release (8). The pharmacological and electrophysiological properties of this channel have been reproduced in yeast and human cell models of apoptosis $(37,38)$. The channel conductance ( 3.3 and $4.5 \mathrm{nS}$ ) was first detected by applying patch-clamp techniques to mitochondria isolated from hematopoietic FL5.12 cell lines, derived from WEHI-3B cells, upon withdrawal of IL-3, and reproduced in outer mitochondrial membranes of yeast expressing human BAX (37). MAC electrophysiological activity is increased by BAX oligomerization in the outer membrane and is prevented by overexpression of BCL-2, but not by cyclosporin A (37). More important, the addition of cytochrome $c$ interferes with the largest conductance state of this voltage-dependent channel, estimated to be $\sim 3.0-4.0 \mathrm{~nm}$ in diameter $(37,39)$. The molecular identification of MAC has yet to be fully determined. Nonetheless, there is considerable evidence that oligomeric BAX/BAX, BAX/BAK or/and BAK/ BAK are components of MAC $(8,39)$.

Mitochondrial membranes change their lipid composition under various apoptotic stimuli $(1,6)$. Recently, a lipid channel composed of the lipid ceramide and named ceramide channel has also been implicated in apoptogenic factor release from mitochondria (9). Ceramides differ from other lipids in that they can form intermolecular hydrogen bonds to produce columns of ceramide residues. Such columns form ceramide channels with multiple conductance states and capable of releasing proteins of up to $60 \mathrm{kDa}$ (9). The specificity of such lipid channels is clearly very limited and, again, awaits convincing evidence for its real role during physiological apoptosis.

\section{Role of caspases in early and delayed mitochondrial dysfunction}

Caspases may be involved both in the earlier events of apoptosis, such as cyto- chrome $c$ release, and in the delayed mitochondrial events that include loss of mitochondrial transmembrane potential and inhibition of electron flow in the respiratory chain (40). Previous studies have shown that incubation of isolated mitochondria with recombinant human caspases promotes membrane permeabilization and the release of cytochrome $c$ and Smac/Diablo into the cytosol $(41,42)$. Uncleaved forms of caspase- 2 efficiently insert into mitochondrial membranes and release cytochrome $c$ bound to anionic phospholipid cardiolipin (42). Similarly, caspase- 3 enters the intermembrane space and cleaves the $75-\mathrm{kDa}$ subunit (NDUF1) of complex I of the electron transport chain of isolated mitochondria (43). Electron transport by complexes I and II is reduced by 88 and $94 \%$, respectively. However, the treatment does not affect oxygen consumption by complex IV (43). Interestingly, treatment with z-VAD-fmk, a pancaspase inhibitor, preserved electron transport chain functionality but failed to inhibit cytochrome $c$ release. Additional studies on intact cells and isolated mitochondria have also shown that $z$-VAD-fmk was able to inhibit the release of Smac/Diablo, HtrA2/ Omi, AIF, and Endo G, but could not inhibit the release of cytochrome $c$ (28). These results agree with a recent study (44) that used single cell analysis to show that cytochrome $c$ release is independent of caspase- 3 activity, but that active caspase- 3 is required for the sustained loss of the mitochondrial transmembrane potential.

A recent study by Lakhani and colleagues (16) has extended the major controversies about the role of executioner caspases in mitochondrial homeostasis. These investigators generated double knockout mice for two highly related effector caspases, caspase-3 and -7. Embryonic fibroblasts and thymocytes derived from mice lacking both enzymes exhibited resistance to drugs that induce the intrinsic (mitochondrial) and extrinsic (membrane death receptor) pathways 
to apoptosis (16). In all conditions studied, the cells displayed a pronounced delay in cytochrome $c$ release and translocation of BAX to the outer membrane. The mitochondrial membrane potential was unaffected. Overall, the results of this study led the authors to conclude that caspase- 3 and -7 are important mediators for mitochondrial events in apoptosis.

CD95 (Fas)- and TNF-mediated apoptosis occurs due to a cascade of morphological and biochemical events that include receptor membrane internalization, death-inducing signaling complex assembly and autoproteolytic cleavage of caspase- 8 inside death-signaling vesicles (45). Activated caspase- 8 can either activate executioner caspase- 3 , or cleave BID, generating tBID, that moves to mitochondria, promoting cytochrome $c$ release (45). Increasing evidence now indicates that an increase of endocytic vacuoles is involved not only in receptor internalization (45), but also in Golgi and mitochondrial intercommunication (46). This provides further support for a model in which caspase-mediated cleavage of mitochondrial proteins could be involved in mitochondrial dysfunction and in the regulation of mitochondrially mediated cell death.

The search for new caspase substrates represents a remarkable challenge and depends largely on biochemical methods such as gel electrophoresis, chromatography and mass spectrometry. Usually, this work can take years. Thus, bioinformatic tools for a quickly search of internal cleavage sites in protein sequences represent an innovative approach. CaSPredictor is a software that uses a novel methodology for characterizing cleavage sites in protein sequences (47). This program uses a scoring scheme that incorporates the position-dependent amino acid at the caspase-pentapeptide cleavage site and the position-independent proline (P), glutamic acid (E), serine (S), and threonine (T) (PEST) amino acids, Glu or Asp (D/E), Asn $(\mathrm{N})$ and Glu (Q) at the right and left flanking of an aspartate residue within a 35amino acid extension. Moreover, the algorithm uses a BLOSUM 62 substitution matrix to find biological similarity between amino acids not annotated in its database (47). The prediction accuracy of CaSPredictor was estimated at $83 \%$, as assessed by ROC analysis. In a large-scale analysis, we identified 1600 predicted caspase substrates with a score $>0.57$, with $60 \%$ sensitivity and $97 \%$ specificity (47).

Given that most transport systems are involved in the translocation of ions, metabolites and proteins across mitochondrial compartments, our investigation attempted to identify those proteins in the ion channel family, $\mathrm{ABC}$ transporter families and the translocase in the inner (Tim) and outer (Tom) mitochondrial membrane family with potential cleavage sites for caspases (Table 1). Some protein candidates are structurally and functionally linked to apoptosis via TNF receptor-associated protein, Fas-associated protein with a death domain and CARD. These observations raised unexplored possibilities that their cleavage may be relevant to diverse mitochondrial dysfunctions, including membrane permeabilization and pore formation, since some of them have the ability to bind and transport proteins across membranes.

The plasma membrane $\mathrm{Ca}^{2+}$ pump (PMCA), $\mathrm{Na}^{+} / \mathrm{Ca}^{2+}$ exchanger (NCX) and $\mathrm{H}^{+} / \mathrm{Ca}^{2+}$ uniporter operate in $\mathrm{Ca}^{2+}$ extrusion and control neuronal cell death $(48,49)$. Proteolytic cleavage and inactivation of these plasma membrane proteins has been demonstrated in two neuronal cell death models induced by prolonged overstimulation of the glutamate receptor subtypes (NMDA and AMPA) that lead to $\mathrm{Ca}^{2+}$ and $\mathrm{Na}^{+}$influx and overload in neurons $(48,49)$. The AMPA receptor is an example of several receptor families that are regulated physiologically by caspases (50). PMCA is cleaved and inactivated by caspases in neurons undergoing excitotoxicity (48). Expression of mu- 
Table 1. Identification, structural properties and localization of cation and anion channels, ATP-binding cassette (ABC) transporters and the translocases in the inner (Tim) and outer (Tom) mitochondrial membrane family containing potential cleavage sites for caspases and the apoptosis interaction domain.

\begin{tabular}{|c|c|c|c|c|}
\hline Protein (GeneBank ID) & Cleavage site & Cellular localization & Domain & $\begin{array}{l}\text { Apoptosis interaction } \\
\text { domain }\end{array}$ \\
\hline $\begin{array}{l}\text { Voltage-dependent calcium channel } \alpha 1 G \text { subunit } \\
\text { (AAD29401) }\end{array}$ & ${ }^{1221}$ ESQDV & Plasma membrane & Calcium channel & No \\
\hline Neuronal calcium channel $\alpha 1 \mathrm{~A}$ subunit (AAB61613) & ${ }^{2421} \mathrm{DEADG}$ & Plasma membrane & Calcium channel & No \\
\hline $\begin{array}{l}\text { Voltage-dependent L-type channel, B-1 subunit, } \\
\text { CAB1 (Q06421) }\end{array}$ & ${ }^{498}$ DTFDA & Cytoplasm & Calcium channel & Ankyrin \\
\hline $\begin{array}{l}\text { Chloride intracellular channel protein } 4, \mathrm{mtCLIC} \text {, } \\
\text { (CAH70045.1) }\end{array}$ & 161DEIDE & Mitochondrial & Chloride channel & CARD-8 \\
\hline $\begin{array}{l}\text { Potassium voltage-gated channel, subfamily G, KCNJ7 } \\
\text { (CAl23445.1) }\end{array}$ & ${ }^{187}$ DALDS & Mitochondrial & K Tetra & No \\
\hline Potassium channel, subfamily T, Kca4.1 (Q5JUK3) & ${ }^{845}$ DNLDS & Plasma membrane & K Tetra & No \\
\hline $\begin{array}{l}\text { ABC transporter (ABCA1), cholesterol and phospholipid } \\
\text { efflux (O95477) }\end{array}$ & ${ }^{1292 D P N D S}$ & Plasma membrane & $\mathrm{ABC}$ transporter & FADD \\
\hline $\begin{array}{l}\text { ABC transporter (ABCA3), chemical and metabolite } \\
\text { efflux (Q99758) }\end{array}$ & ${ }^{197}$ TSPDG & Plasma membrane & $\mathrm{ABC}$ transporter & ICAD \\
\hline $\begin{array}{l}\text { ABC transporter (ALDP/ABCD1), adrenoleukodystrophy } \\
\text { protein (P33897) }\end{array}$ & ${ }^{358}$ SESDA & Plasma membrane & $\mathrm{ABC}$ transporter & CARD-4 \\
\hline $\begin{array}{l}\text { ABC transporter (hALDR/ABCD2), adrenoleukodystrophy } \\
\text { protein (Q99758) }\end{array}$ & ${ }^{565}$ DSVDD & Plasma membrane & $\mathrm{ABC}$ transporter & CARD-4 \\
\hline $\begin{array}{l}\text { Cystic fibrosis transmembrane conductance regulator } \\
\text { (CFTR/ABCC7) (P13569) }\end{array}$ & 1272VSWDS & Plasma membrane & $\mathrm{ABC}$ transporter & TRAF-3 \\
\hline $\begin{array}{l}\text { ABC transporter ( } A B C C 1) \text {, multidrug resistance- } \\
\text { associated protein } 1(\mathrm{P} 33527)\end{array}$ & ${ }^{1081}$ DTVDS & Plasma membrane & $\mathrm{ABC}$ transporter & TRAF-3,-5 \\
\hline $\begin{array}{l}\text { ABC transporter }(A B C B 7) \text {, iron transport to mitochondria } \\
(O 75027)\end{array}$ & ${ }^{637}$ SSLDS & Mitochondrial & $A B C$ transporter & v-FLIP \\
\hline $\begin{array}{l}\text { Multidrug protein resistance-3 (ABCB4), P-glycoprotein-3 } \\
\text { (P21439) }\end{array}$ & ${ }^{683}$ VETDG & Plasma membrane & $\mathrm{ABC}$ transporter & CARD-4 \\
\hline $\begin{array}{l}\text { Translocase of outer mitochondrial membrane } 22 \mathrm{kDa} \text {, } \\
\text { Tom22 (Q9NS69) }\end{array}$ & ${ }^{184} \mathrm{EELDE}$ & Mitochondrial & No & No \\
\hline $\begin{array}{l}\text { Translocase of outer mitochondrial membrane } 70 \mathrm{kDa} \text {, } \\
\text { Tom70 (O94826) }\end{array}$ & ${ }^{538}$ IEIDN & Mitochondrial & Tetratricopeptide & No \\
\hline $\begin{array}{l}\text { Translocase of inner mitochondrial membrane } 50 \mathrm{kDa} \text {, } \\
\text { Tim50 (AAT01208.1) }\end{array}$ & ${ }^{203}$ DEFDN & Mitochondrial & Ctd phosphatase & No \\
\hline
\end{tabular}

These proteins were identified in a large-scale analysis of 9800 human proteins using the CasPredictor software. Database available at http:// icb.usp.br/ farmaco/jose. Domains for caspase-protein substrate interactions are indicated in the table. No means that interaction domain has not been found. $C A R D=$ caspase recruitment domain; FADD = Fas-associated protein with a death domain: ICAD = inhibitor of caspase-activated Dnase; TRAF = TNF receptor-associated protein: v-FLIP = viral FLICE inhibitory protein. 
tant forms of human PMCA4 that lack the caspase cleavage site prevents the delayed rise of $\mathrm{Ca}^{2+}$ or delayed $\mathrm{Ca}^{2+}$ deregulation (48). Bano and colleagues (49) have also shown that both calpains and caspases can cleave NCX during excitotoxicity in neuronal cell lines. The broad-spectrum caspase inhibitor z-VAD-fmk moderately reduced the caspase-mediated cleavage of NCX3 and delayed $\mathrm{Ca}^{2+}$ deregulation that causes necrotic cell death (49). Therefore, caspases appear to be involved in the control of transmembrane $\mathrm{Ca}^{2+}$ channels and pumps and indirectly in the increase of intracellular $\mathrm{Ca}^{2+}$ pools that may precede the opening of mitochondrial death decision pores.

The superfamily of ATP-binding cassette $(A B C)$ transporter proteins consists of efflux pumps that have important roles in transporting a diverse group of toxicants including lipophilic cationic, anionic, and neutrally charged drugs, peroxidation products and antigenic peptides $(51,52)$. ABC transporters are localized in the plasma membrane, endoplasmic reticulum and in organelles, including peroxisomes and mitochondria. Five mammalian mitochondrial $\mathrm{ABC}$ transporters have been described based on the presence of mitochondrial targeting presequences $(51,52)$. Members of this family are involved in the export of iron-sulfurcontaining proteins such as apoproteins from the mitochondrial matrix to the cytosol (51). Deletions of mitochondrial ABC transporters disturb iron homeostasis and are lethal (53). At present, one study (54) has shown that the proteolytic cleavage of ABCA1, a transmembrane transporter involved in cytosolic cholesterol efflux by calpain, increases its activity. An uncleavable form of ABCA1 displayed a 4-fold increase in the efflux of cholesterol, which prevented macrophage cell death due to cholesterol overload (54). We noted that calpain and caspases have cleavage sites within the same PEST sequence (Table 1). This raises the chance that both proteases could regulate the pumping activity of $\mathrm{ABC}$ transporters.

Mitochondrial chloride intracellular channel protein 4 (mtCLIC) belongs to a CLIC family of soluble globular proteins that can form ion channels in organelles and plasma membranes similar to bacterial toxins, annexins and $\mathrm{BCL}-\mathrm{x}_{\mathrm{L}}$ (55). It has been shown that mtCLIC protein 4 is up-regulated during the cell death response to cytotoxic agents and DNA damage. Overexpression of the mtCLIC gene is sufficient to induce keratinocyte cell death, which is associated with the expression of $\mathrm{p} 53$, loss of mitochondrial membrane potential and cytochrome $c$ release (54). More importantly, cell death is inhibited by z-VAD-fmk, a pancaspase inhibitor (55).

The translocase of the inner (Tim complex) and outer (Tom complex) mitochondrial membranes is composed of large and small proteins that display an intrinsic capacity to bind and transfer polypeptides into mitochondrial compartments (56). They contain water-filled pores that mediate translocation of proteins tagged with presequences that act as bipartite sorting signals. These $\mathrm{N}$ terminal sorting signals form amphipathic helices with a hydrophobic and positively charged side. The sequences are first recognized by receptors and targeted to the protein-conducting channel (Tom proteins). This translocation mechanism depends on both ATP and the mitochondrial membrane potential (56).

The role the Tim 23 complex for translocation of AIF, Endo G, Smac/Diablo, and HtrA2/Omi into the intermembrane mitochondrial space has been demonstrated (56). Furthermore, a study with yeast mitochondria has shown that perturbations of Tim 23 conductance activity with synthetic peptides and specific antibodies cause matrix swelling and ultimately cytochrome $c$ release (57). Tim50 is a subunit of the Tim 23 complex (56). It has been demonstrated that clones of human cells and zebrafish embryos lacking the Tim50 gene undergo mitochondrial dys- 
function and rapid apoptosis (58). Tom20 and Tom 22 have a negatively charged $\mathrm{N}$ terminal region exposed to the cytosol that contains a putative cleavage site for caspases. This region coordinates the recognition and translocation of many nuclear proteins into mitochondria (58). Interestingly, deletion of the Tom22 gene is lethal (56). A complex of approximately $400 \mathrm{kDa}$ containing Tom20, Tom40 and Tom70 operates in the translocation of apocytochrome $c$ inside the intermembrane space, where it incorporates the heme co-factor and is released as holo-cytochrome $c$ (56). The possible consequences of Tom 22 cleavage by caspases are numerous. For example, it could interrupt cytochrome $c$ import causing electron transport interruption. In conclusion, Tim/ Tom complexes appear to be interesting pathways for apoptogenic factor release. Future studies are needed to challenge this hypothesis and to test how caspases can affect protein translocation across mitochondrial membranes.

\section{Outer membrane rupture and mitochondrial remodeling}

The occurrence of swelling is common in the mitochondria of the cells committed to die, as a result of inner membrane permeability transition $(20,21)$. Since the surface of the inner membrane is greater than the surface of the outer membrane, the swelling forces the expansion of the inner membrane to the cytoplasm, causing the outer membrane to rupture (10). Korsmeyer's group has also described the morphological features of degenerating mitochondria treated with tBID and crista ultrastructural remodeling using high-voltage electron microscopy and tomography (59). The authors proposed that tBID is necessary for mitochondrial remodeling and further mobilization of cytochrome $c(\sim 85 \%)$ that is retained in mitochondrial crista stores. Based on their crista remodeling, the mitochondria were classi- fied as class I (normal) or class II, III, and IV (59). Class II mitochondria with highly interconnected and condensed cristae occur after 2-5 min in cells treated with tBID, TNF and Fas and several intrinsic death stimuli including thapsigargin, tunicamycin and brefeldin A (59). Class III mitochondria are swollen and their outer membrane are ruptured. This is comparable to the type II mitochondrial profile described by Sesso and colleagues (10).

Finally, fusion and fission (division) are two morphologically and physiologically opposite processes within mitochondrial dynamics. It has been demonstrated that perturbations in these processes result in mitochondrial outer membrane permeabilization and release of apoptogenic factors and apoptosis (60). Cytosolic GTPases of the dynamin family, optic atrophy 1 and mitofusins 1 and 2 , are required for mitochondrial fusion, while dynamin-related protein 1 and Fis 1 are required for mitochondrial fission (60).

The present overview suggests that numerous biomolecules that are produced by biochemical and bioenergetic reactions inside and outside the mitochondria, and regulators and effectors of apoptosis play a role in the coordination of events involved in mitochondrial membrane permeabilization and the release of cytochrome $c$, Smac/ Diablo, HtrA2/Omi and AIF. An integrated model is depicted in Figure 1. Permeabilization of the inner or outer membrane must be accompanied by exchange of cations, like $\mathrm{Ca}^{2+}, \mathrm{K}^{+}$, and $\mathrm{Na}^{+}$, and metabolites that have an important impact on mitochondrial volume, bioenergetic reactions and the mitochondrial membrane potential. The opening and release of apoptogenic proteins occur via one of the death decision pores, namely the PTP formed in the inner mitochondrial membrane or a proteolipid pore formed by the MOMP. The molecular components of PTP and MOMP pore are variable or remain to be determined. However, cytosolic and mitochondrial proteins, including VDAC, 
ANT and pro-apoptotic proteins tBID, BAX and BAK, may participate in their assembly and operation. The MAC and a ceramide lipid pore may also be involved in the release of the apoptogenic factors. Nonetheless, the simple possibility that apoptogenic factor release occurs through a nonspecific mitochondrial outer membrane rupture also remains valid. Our understanding of the critical role of caspases in mitochondrial dysfunction is based on the experiments using recombinant caspases, their peptide inhibitors and mice deficient in both caspase- 3 and caspase-7. Few components of the mitochondrial machinery have been described as caspase substrates. One study has shown that caspase-3 can enter the mitochondria and cleave NDUF1, a component of complex I of the respiratory chain. It remains unclear how a cytosolic protease can cross both mitochondrial membranes to cleave a matrix-exposed subunit embedded within complex I. However, biochemical analysis has demonstrated that pro-caspase and active caspase $(-3,-7,-8$, and -9$)$ can partially co-localize in the outer membrane or are released into mitochondria via "death signaling vesicles" stimulated by TNF and CD95/Fas $(45,46)$. In this scenario, it may be possible that caspases, especially the apical

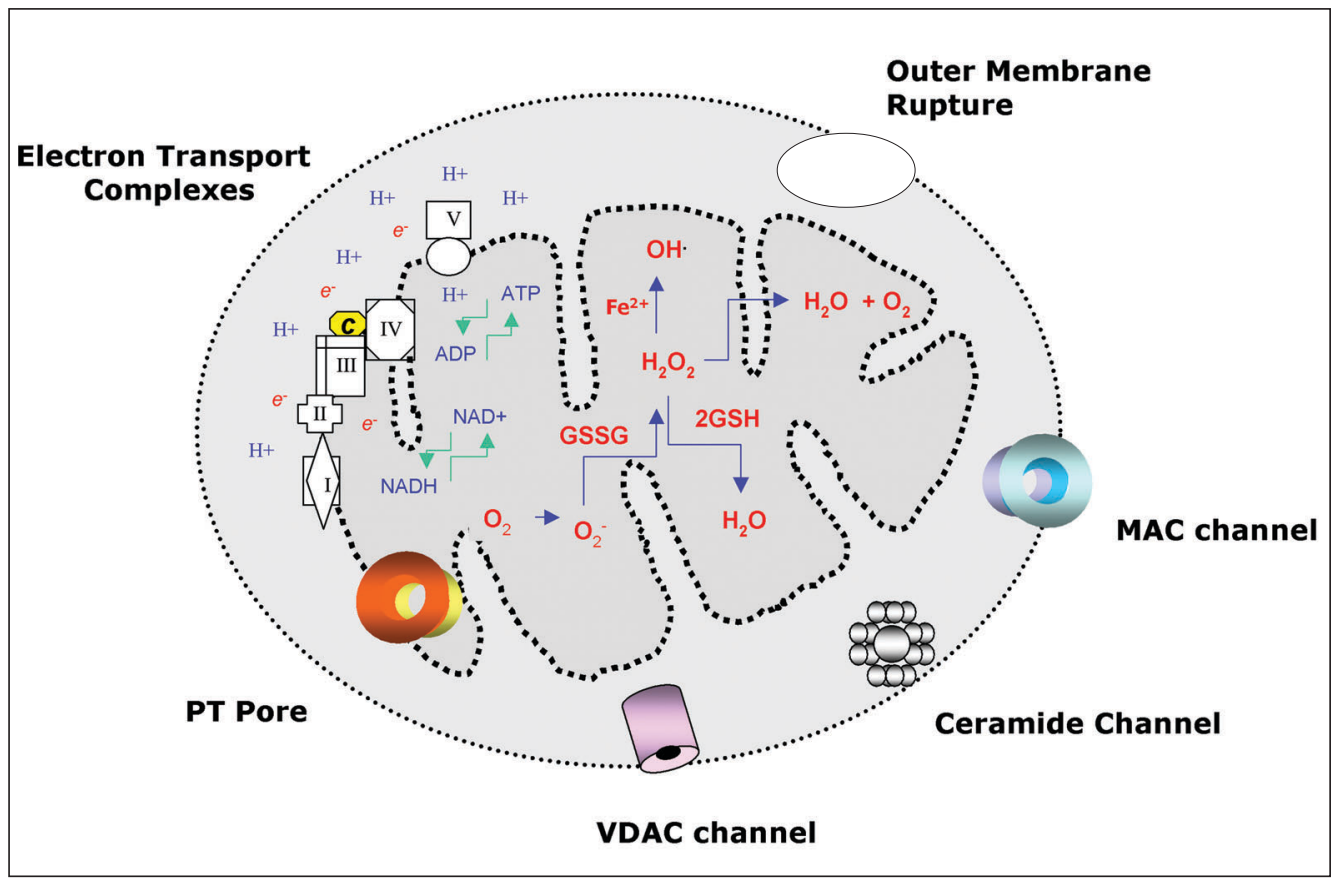

Figure 1. Schematic representation of mitochondrial death decision pores with the biochemical ability to promote the inner and outer mitochondrial membrane permeabilization and the release of apoptogenic factors: cytochrome $c$, apoptosis-inducing factor, Smac/Diablo, HtrA2/Omi, and endonuclease G. The four-channel/pore-forming complexes described are: I) a large conductance pore-forming complex, named permeability transition pore (PTP) that is formed in the inner mitochondrial membrane in response to radical oxygen species overproduction during bioenergetic reactions and $\mathrm{Ca}^{2+}$ overload; II) a proteolipid pore formed in response to outer mitochondrial membrane permeabilization caused by oligomerized forms of BAX and BAK after their activation by tBID and named mitochondrial apoptosis-inducing channel (MAC); III) a lipid channel formed by the lipid ceramide, and IV) voltage-dependent anion channel (VDAC). Outer membrane rupture could also act as an alternative route for apoptogenic factor release. Various components of the channel- and pore-forming subunits may be cleaved by caspases (see Table 1). Their cleavage might cause changes in their structural organization and a switch to a nonspecific channel or pore with different conductance or selectivity, or turn off the channel or transport system. The validity of this mechanistic model needs to be examined experimentally. 
ones, gain access to mitochondria via alteration in endocytic traffic. Using a computational program, we found that some members of cytoplasmic and mitochondrial ABC transporters and regulatory components of Tim and Tom complexes as well as some subunits of the plasma membrane and mitochondrial membrane channels for $\mathrm{Cl}^{-}$and $\mathrm{K}^{+}$and $\mathrm{Ca}^{2+}$ are potential caspase substrates. Their cleavage could cause changes in their structure and the switch to a nonspecific channel or pore with different conductance, selectivity or functional state. The validity of this mechanistic model needs to be examined experimentally. Future studies for the characterization of the molecular components and of the mechanistic basis by which the mitochondrial death decision pores operate will certainly help the design of new therapies to amplify or block the release of apoptogenic factors.

\section{Acknowledgments}

The authors are grateful to Alicia Kowaltowski (Departamento de Bioquímica, IQ-USP), Gustavo Pessini Amarante-Mendes (Departamento de Imunologia, ICB-USP), Mauro Degli Esposti (Faculty of Life Sciences, University of Manchester, UK), and Tatiana Rostovtseva (Laboratory of Physical and Structural Biology, NICHD-NIH) for expert reviews of the manuscript and helpful comments.

\section{References}

1. Lemasters JJ. Dying a thousand deaths: redundant pathways from different organelles to apoptosis and necrosis. Gastroenterology 2005; 129: 351-360.

2. Chipuk JE, Bouchier-Hayes L, Green DR. Mitochondrial outer membrane permeabilization during apoptosis: the innocent bystander scenario. Cell Death Differ 2006; 13: 1396-1402.

3. Vander Heiden MG, Thompson CB. Bcl-2 proteins: regulators of apoptosis or of mitochondrial homeostasis? Nat Cell Biol 1999; 1: E209-E216.

4. Scorrano L, Korsmeyer SJ. Mechanisms of cytochrome c release by proapoptotic BCL-2 family members. Biochem Biophys Res Commun 2003; 304: 437-444.

5. Breckenridge DG, Xue D. Regulation of mitochondrial membrane permeabilization by BCL-2 family proteins and caspases. Curr Opin Cell Biol 2004; 16: 647-652.

6. Lucken-Ardjomande S, Martinou JC. Newcomers in the process of mitochondrial permeabilization. J Cell Sci 2005; 118: 473-483.

7. Zoratti M, Szabo I, De Marchi U. Mitochondrial permeability transitions: how many doors to the house? Biochim Biophys Acta 2005; 1706: 40-52.

8. Dejean LM, Martinez-Caballero S, Kinnally KW. Is MAC the knife that cuts cytochrome c from mitochondria during apoptosis? Cell Death Differ 2006; 13: 1387-1395.

9. Siskind LJ. Mitochondrial ceramide and the induction of apoptosis. $J$ Bioenerg Biomembr 2005; 37: 143-153.

10. Sesso A, Marques MM, Monteiro MM, Schumacher RI, Colquhoun A, Belizario J, et al. Morphology of mitochondrial permeability transition: morphometric volumetry in apoptotic cells. Anat Rec A Discov Mol Cell Evol Biol 2004; 281: 1337-1351.

11. Magder S. Reactive oxygen species: toxic molecules or spark of life? Crit Care 2006; 10: 208.

12. Storz P. Reactive oxygen species-mediated mitochondria-to-nucleus signaling: a key to aging and radical-caused diseases. Sci STKE
2006; 2006: re3.

13. Shi Y. Caspase activation, inhibition, and reactivation: a mechanism view. Protein Sci 2004; 13: 1979-1987.

14. Fischer $U$, Janicke RU, Schulze-Osthoff $K$. Many cuts to ruin: a comprehensive update of caspase substrates. Cell Death Differ 2003; 10: 76-100.

15. Marzo I, Susin SA, Petit PX, Ravagnan L, Brenner C, Larochette N, et al. Caspases disrupt mitochondrial membrane barrier function. FEBS Lett 1998; 427: 198-202.

16. Lakhani SA, Masud A, Kuida K, Porter GA Jr, Booth CJ, Mehal WZ, et al. Caspases 3 and 7: key mediators of mitochondrial events of apoptosis. Science 2006; 311: 847-851.

17. Orrenius S, Zhivotovsky B, Nicotera P. Regulation of cell death: the calcium-apoptosis link. Nat Rev Mol Cell Biol 2003; 4: 552-565.

18. Waring P. Redox active calcium ion channels and cell death. Arch Biochem Biophys 2005; 434: 33-42.

19. Kowaltowski AJ, Castilho RF, Vercesi AE. Mitochondrial permeability transition and oxidative stress. FEBS Lett 2001; 495: 12-15.

20. Skulachev VP. Bioenergetic aspects of apoptosis, necrosis and mitoptosis. Apoptosis 2006; 11: 473-485.

21. Bernardi P, Krauskopf A, Basso E, Petronilli V, Blachly-Dyson E, Di $\mathrm{LF}$, et al. The mitochondrial permeability transition from in vitro artifact to disease target. FEBS J 2006; 273: 2077-2099.

22. Launay S, Hermine O, Fontenay M, Kroemer G, Solary E, Garrido C. Vital functions for lethal caspases. Oncogene 2005; 24: 5137-5148.

23. Cain K, Bratton SB, Cohen GM. The Apaf-1 apoptosome: a large caspase-activating complex. Biochimie 2002; 84: 203-214.

24. Vaux DL, Silke J. IAPs, RINGs and ubiquitylation. Nat Rev Mol Cell Biol 2005; 6: 287-297.

25. Springs SL, Diavolitsis VM, Goodhouse J, McLendon GL. The kinetics of translocation of Smac/DIABLO from the mitochondria to the cytosol in HeLa cells. J Biol Chem 2002; 277: 45715-45718.

26. Rehm M, Dussmann H, Prehn JH. Real-time single cell analysis of 
Smac/DIABLO release during apoptosis. J Cell Biol 2003; 162: 1031-1043.

27. Uren RT, Dewson G, Bonzon C, Lithgow T, Newmeyer DD, Kluck RM. Mitochondrial release of pro-apoptotic proteins: electrostatic interactions can hold cytochrome c but not Smac/DIABLO to mitochondrial membranes. J Biol Chem 2005; 280: 2266-2274.

28. Arnoult D, Gaume B, Karbowski M, Sharpe JC, Cecconi F, Youle RJ. Mitochondrial release of AIF and EndoG requires caspase activation downstream of Bax/Bak-mediated permeabilization. EMBO $J$ 2003; 22: 4385-4399.

29. Scorrano L, Oakes SA, Opferman JT, Cheng EH, Sorcinelli MD, Pozzan $\mathrm{T}$, et al. BAX and BAK regulation of endoplasmic reticulum $\mathrm{Ca}^{2+}$ : a control point for apoptosis. Science 2003; 300: 135-139.

30. Colombini M. VDAC: the channel at the interface between mitochondria and the cytosol. Mol Cell Biochem 2004; 256-257: 107-115.

31. Lemasters JJ, Holmuhamedov E. Voltage-dependent anion channel (VDAC) as mitochondrial governator - thinking outside the box. Biochim Biophys Acta 2006; 1762: 181-190.

32. Rostovtseva TK, Antonsson B, Suzuki M, Youle RJ, Colombini M, Bezrukov SM. Bid, but not Bax, regulates VDAC channels. J Biol Chem 2004; 279: 13575-13583.

33. Shimizu S, Narita M, Tsujimoto Y. Bcl-2 family proteins regulate the release of apoptogenic cytochrome $\mathrm{c}$ by the mitochondrial channel VDAC. Nature 1999; 399: 483-487.

34. Crompton M, Barksby E, Johnson N, Capano M. Mitochondrial intermembrane junctional complexes and their involvement in cell death. Biochimie 2002; 84: 143-152.

35. Rostovtseva TK, Tan W, Colombini M. On the role of VDAC in apoptosis: fact and fiction. J Bioenerg Biomembr 2005; 37: 129-142.

36. Abu-Hamad S, Sivan S, Shoshan-Barmatz V. The expression level of the voltage-dependent anion channel controls life and death of the cell. Proc Natl Acad Sci U S A 2006; 103: 5787-5792.

37. Pavlov EV, Priault M, Pietkiewicz D, Cheng EH, Antonsson B, Manon S, et al. A novel, high conductance channel of mitochondria linked to apoptosis in mammalian cells and Bax expression in yeast. $J$ Cell Biol 2001; 155: 725-731.

38. Guo L, Pietkiewicz D, Pavlov EV, Grigoriev SM, Kasianowicz JJ, Dejean LM, et al. Effects of cytochrome $c$ on the mitochondrial apoptosis-induced channel MAC. Am J Physiol Cell Physiol 2004; 286: C1109-C1117.

39. Guihard G, Bellot G, Moreau C, Pradal G, Ferry N, Thomy R, et al. The mitochondrial apoptosis-induced channel (MAC) corresponds to a late apoptotic event. J Biol Chem 2004; 279: 46542-46550.

40. Ricci JE, Waterhouse N, Green DR. Mitochondrial functions during cell death, a complex (I-V) dilemma. Cell Death Differ 2003; 10: 488492.

41. Lassus $P$, Opitz-Araya X, Lazebnik Y. Requirement for caspase-2 in stress-induced apoptosis before mitochondrial permeabilization. Science 2002; 297: 1352-1354.

42. Enoksson M, Robertson JD, Gogvadze V, Bu P, Kropotov A, Zhivotovsky B, et al. Caspase-2 permeabilizes the outer mitochondrial membrane and disrupts the binding of cytochrome $c$ to anionic phospholipids. J Biol Chem 2004; 279: 49575-49578.

43. Ricci JE, Munoz-Pinedo C, Fitzgerald P, Bailly-Maitre B, Perkins GA, Yadava N, et al. Disruption of mitochondrial function during apoptosis is mediated by caspase cleavage of the p75 subunit of complex I of the electron transport chain. Cell 2004; 117: 773-786. 44. Waterhouse NJ, Sedelies KA, Sutton VR, Pinkoski MJ, Thia KY, Johnstone R, et al. Functional dissociation of DeltaPsim and cytochrome $\mathrm{c}$ release defines the contribution of mitochondria upstream of caspase activation during granzyme B-induced apoptosis. Cell Death Differ 2006; 13: 607-618.

45. Lee KH, Feig C, Tchikov V, Schickel R, Hallas C, Schutze S, et al. The role of receptor internalization in CD95 signaling. EMBO $J$ 2006; 25: 1009-1023.

46. Ouasti S, Matarrese P, Paddon R, Khosravi-Far R, Sorice M, Tinari $\mathrm{A}$, et al. Death receptor ligation triggers membrane scrambling between Golgi and mitochondria. Cell Death Differ 2006; 14: 456-461.

47. Garay-Malpartida HM, Occhiucci JM, Alves J, Belizario JE. CaSPredictor: a new computer-based tool for caspase substrate prediction. Bioinformatics 2005; 21 (Suppl 1): i169-i176.

48. Schwab BL, Guerini D, Didszun C, Bano D, Ferrando-May E, Fava $\mathrm{E}$, et al. Cleavage of plasma membrane calcium pumps by caspases: a link between apoptosis and necrosis. Cell Death Differ 2002; 9: 818-831.

49. Bano D, Young KW, Guerin CJ, Lefeuvre R, Rothwell NJ, Naldini L, et al. Cleavage of the plasma membrane $\mathrm{Na}+/ \mathrm{Ca} 2+$ exchanger in excitotoxicity. Cell 2005; 120: 275-285.

50. Bredesen DE, Mehlen $\mathrm{P}$, Rabizadeh $\mathrm{S}$. Receptors that mediate cellular dependence. Cell Death Differ 2005; 12: 1031-1043.

51. Lill R, Kispal G. Mitochondrial ABC transporters. Res Microbiol 2001; 152: 331-340.

52. Higgins $C F$, Linton KJ. The ATP switch model for ABC transporters. Nat Struct Mol Biol 2004; 11: 918-926.

53. Senbongi $H$, Ling $F$, Shibata $T$. A mutation in a mitochondrial $A B C$ transporter results in mitochondrial dysfunction through oxidative damage of mitochondrial DNA. Mol Gen Genet 1999; 262: 426-436.

54. Wang N, Chen W, Linsel-Nitschke P, Martinez LO, Agerholm-Larsen $B$, Silver DL, et al. A PEST sequence in ABCA1 regulates degradation by calpain protease and stabilization of ABCA1 by apoA-I. J Clin Invest 2003; 111: 99-107.

55. Fernandez-Salas E, Suh KS, Speransky VV, Bowers WL, Levy JM, Adams $\mathrm{T}$, et al. mtCLIC/CLIC4, an organellular chloride channel protein, is increased by DNA damage and participates in the apoptotic response to p53. Mol Cell Biol 2002; 22: 3610-3620.

56. Herrmann JM, Hell K. Chopped, trapped or tacked - protein translocation into the IMS of mitochondria. Trends Biochem Sci 2005; 30: 205-211.

57. Guo Y, Cheong N, Zhang Z, De Rose R, Deng Y, Farber SA, et al. Tim50, a component of the mitochondrial translocator, regulates mitochondrial integrity and cell death. J Biol Chem 2004; 279: 2481324825.

58. Nargang FE, Rapaport D, Ritzel RG, Neupert W, Lill R. Role of the negative charges in the cytosolic domain of TOM22 in the import of precursor proteins into mitochondria. Mol Cell Biol 1998; 18: 31733181.

59. Scorrano L, Ashiya M, Buttle K, Weiler S, Oakes SA, Mannella CA, et al. A distinct pathway remodels mitochondrial cristae and mobilizes cytochrome c during apoptosis. Dev Cell 2002; 2: 55-67.

60. Martinou JC, Youle RJ. Which came first, the cytochrome $c$ release or the mitochondrial fission? Cell Death Differ 2006; 13: 1291-1295. 